BNL - 66235

CAP-255-Muon-99

\title{
An ionization Cooling Channel for Muon Beams Based on Alternating Solenoids
}

\author{
Juan C. Gallardo, Richard C. Fernow, Harold G. Kirk, \\ Robert B.Palmer \\ Brookhaven National Laboratory \\ Paul Lebrun, Alfred Moretti, Alvin T. Tollestrup \\ Fermilab \\ Daniel M. Kaplan \\ IIT, Chicago, IL \\ and \\ Yasuo Fukui \\ KEK
}

January 1999 


\title{
AN IONIZATION COOLING CHANNEL FOR MUON BEAMS BASED ON ALTERNATING SOLENOIDS*
}

\author{
Juan C. Gallardo, Richard C. Fernow, Harold G. Kirk. Robert B. Palmer, BNL, Lpton NY \\ $\underline{\text { Paul Lebrun }}^{\dagger}$, Alfred Moretti. Alvin V. Tollestrup, FNAL. Batavia. II. \\ Daniel M. Kaplan, IIT. Chicago, IL. \\ Yasuo Fukui, KEK, Tsukuba, Japan
}

\begin{abstract}
The muon collider requires intense, cooled muon bunches to reach the required luminosity. Due to the limited lifetime of the muon, the cooling process must take place very rapidly. Ionization cooling seems to be our only option, given the large emittances of the muon beam from pion decay. However, this ionization cooling method has been found quite difficult to implement in practice. We describe a scheme based on the use of liquid hydrogen absorbers followed by r.f. cavities ("pillbox" or "open iris" type), embedded in a transport lattice based on high field solenoids. These solenoidal fields are reversed periodically in order to suppress the growth of the canonical angular momentum. This channel has been simulated in detail with independent codes, featuring conventional tracking in e.m. fields and detailed simulation of multiple scattering and straggling in the the absorbers and windows. These calculations show that the 15 Tesla lattice cools in $6-D$ phase space by a factor $\approx 2$ over a distance of $20 \mathrm{~m}$.
\end{abstract}

\section{INTRODUCTION}

Ionization cooling of intense muon beams $\left(\approx 2.10^{12}\right.$ particles per bunch) has been recognized as one of the most challenging aspects of the design of a high energy, high intensity Muon Collider[1]. The principle of ionization cooling via deceleration in an absorber followed by re-acceleration in a LINAC, proposed more than 20 years ago[2], is relatively straightforward[1]. However, due to the relarively short muon lifetime, such cooling channels must be very efficient. This entails reaching low values for the $\beta_{\perp}$ function rapidly, and therefore high focusing magnetic fields. The performance of such lattices needs to be verified with accurate simulations, with complete field maps and detailed simulation of the straggling and multiple scattering in the absorbers and foils. Three distinct computer codes were used to study the performance of this channel: (i) a special version of PARMELA[3] in which multiple scattering was simulated using the Gaussian approximation; (ii) ICOOL[4]; (iii) an extended version of the GEANT3 package[5], DPGeant, featuring higher numerical accuracy in tracking particles over large distances and full e.m. field

\footnotetext{
- Work suppored by the U.S. Department of Energy.

'Email: lebrun@fnal.gov
}

simulation in the r.f. cavities. Although results presented in this paper have been obtained with DPGeant, these various simulation agree within the expected code accuracy.

\section{DESCRIPTION OF THE 15 TESLA ALTERNATING SOLENOID COOLING CHANNEL}

We here consider one component of a single cooling stage. consisting of the transverse cooling channel. This muon cooler, sketched in figure 1 is based on the use of the lowest $\mathrm{Z}$ material, liquid hydrogen $\left(\mathrm{LH}_{2}\right)$, embedded in a strong (15 T) solenoidal magnetic field. Linacs are inserted between these absorbers, to compensate for the energy loss. This channel has been designed for a nominal momentum of $187 \mathrm{MeV} / \mathrm{c}(\beta=0.87)$ and accepts a transverse normalized emittance of $\approx 1600$. mm mRad, a momentum spread of $\approx 3 \%$ and a bunch length of $\approx 1.5 \mathrm{~cm}$. The linac r.f. frequency is $805 \mathrm{MHz}$.

To provide the longitudinal focusing required to keep this single bunch together these linacs must be separated by short distances ( less than 50 to $75 \mathrm{~cm}$ ). Thus one section (defined as one deceleration in the absorber followed by one LINAC) is approximately $2 \mathrm{~m}$ long, where the muons will lose and gain $\approx 20 \mathrm{MeV}$, leaving the momentum unchanged after one section. Without the absorbers and linacs. the beam would have a constant $(0)$ value for the canonical angular momentum, despite the large fluctuation of the $\beta_{\perp}$ function. However, the presence of the absorbers causes the canonical angular momentum to grow and would lead to severe transverse emittance growth at the end of a channel made of multiple sections. This growth is stopped by reversing the direction of the solenoidal field each time the beam goes though the high $\beta_{\perp}$ points in the lattice. which are located in the middle of the linacs.

The transport in such a channel is far from isochronous: particles at large transverse amplitude have longer path lengths, causing a longitudinal growth as they reach the linac slightly out of phase. It is therefore necessary to start with a bunch where particles at large amplitude have higher momentum, by up to $\approx 20 \mathrm{MeV} / \mathrm{c}$ for a particle at $\approx 3 \sigma$.

For a fixed maximum field $(15 \mathrm{~T})$, the transverse cooling rate decreases as the beam goes though these sections. because this rate is proportional to the transverse emittance. 
The longitudinal emittance grows because of the stochastic processes in the absorbers (energy loss and multiple scattering). In addition, there are higher order effects in the beam optics and the longitudinal/transverse coupling induce longitudinal motions that are are difficult to compensate for. Thus. the length of such a channel has to be limited to approximately $20 \mathrm{~m}$. after which one must go though an emittance exchange section.

The performance of such a channel has been studied for two different lengths of a section: the $2 \mathrm{~m}(1.5 \mathrm{~m})$ section has a $64 \mathrm{~cm}(42 . \mathrm{cm})$ liquid hydrogen absorber and $1.3 \mathrm{~m}$ $(0.98 \mathrm{~m})$ r.f. cavity, respectively. For the short section case, two different configurations of the linac have been simulated:

1. A "pillbox" type cell, $\pi / 2$, with a peak field on axis of $36 \mathrm{MV} / \mathrm{m}$. and 12 identical cells. The beams goes though the walls of each cell, traversing $13125 . \mu \mathrm{m}$ beryllium foil

2. A more conventional open cell cavity runs in $\pi$ mode, where the iris aperture matches the transverse size of the beam (radius $\approx 4.5 \mathrm{~cm}$ at the entrance and exit points of the cavity, and $8 \mathrm{~cm}$ at $\beta_{\perp}$ max). The peak field on axis is $60 \mathrm{MV} / \mathrm{m}$, implying maximum field of $\approx 90 \mathrm{MV} / \mathrm{m}$ on the iris wall.

The lengths of these cavities as well as the phase for the synchronous, on axis particle were kept identical. This synchronous phase[6] was set to $\approx 30$ degrees, so only approximately one half of the voltage is used for acceleration, the linac acts as much as a buncher as an accelerator. Finally, the liquid hydrogen must be contained in a vessel thermally insulated from the linac, this is achieved using thin $(\approx 1.5$ mil) stainless steel window(s).

\section{PERFORMANCE AND ISSUES}

The transverse and longitudinal emittances as function of the distance along the channel are shown in figure 2, for the $1.5 \mathrm{~m}$ long section and for the open-iris cavity. Over a distance of 25 . meters, the channel achieves a cooling of about a factor 2.3 in transverse emittance and a significant longitudinal reheating of about 2.1 . Therefore, the overall 6-D emittance is reduced from $2.210^{9}(\pi \mathrm{mm} \mathrm{mRad})^{3}$ down to $0.86(\pi \mathrm{mm} \mathrm{mRad})^{3}$. Correspondignly, the r.m.s. beam size (radius) in the channel decreases from $1.09 \mathrm{~cm}$ down to $0.7 \mathrm{~cm}$.

Over $20 \mathrm{~m}$, the non-decay loss is found to be less than $1 \%$. For longer channels. this beam loss increases rapidly as the bunch length grows; at the end of the channel, the bucket develops an "alpha" shape, signaling the need to stop cooling transversely and recover control of the longitudinal emittance. Very similar results were obtained with the pillbox cavity. Provided the average net acceleration across the cavity is matched to the energy loss in the absorber and sufficient voltage is applied to ensure re-bunching, the net cooling mert factor of such a channel does not depend on
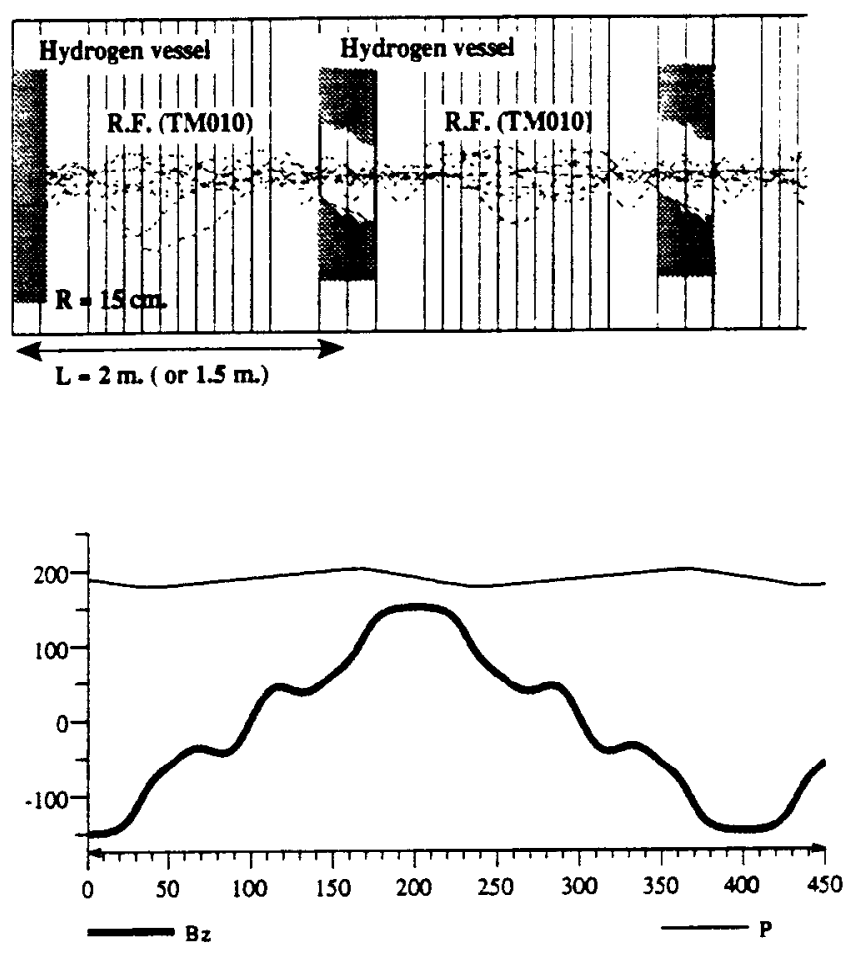

Figure 1: The Alternate Solenoidal muon cooling channel (top). The hashed lines are actual traces of muons obtained via the DPGeant package. Also shown (bottom), with approximately the same horizontal scale, are the longitudinal component of the magnetic field in KG., and the momentum for the synchronous particle $(\mathrm{MeV} / \mathrm{c})$.

the details of the acceleration system. However, the multiple scattering occurring at relatively high $\beta_{\perp}$ in the lattice must remain small, i.e., thin $(\leq 125 . \mu m)$ beryllium windows must be used. The tolerance on the phase $( \pm 3$ degrees is not particularly stringent. The difference in peak field is simply due to the different transit time factors for the two modes ( $\pi$ vs $\pi / 2$.).

The $2 \mathrm{~m}$ long section was found to be slightly worse than the $1.5 \mathrm{~m}$ one. The ratio of $6 \mathrm{D}$ emittances initial over final is about $10 \%$ smaller than for the $1.5 \mathrm{~m}$ long section. In principle, a longer cell period could give us faster transverse cooling time because less longitudinal space is wasted in providing access cooling/shielding equipment between the absorber and the r.f. Longer section section also minimizes multiple scattering in the windows of the $L H_{2}$ vessels. However, the non-relativistic bucket would drift for a longer time in the absorber, resulting in a faster increase of 
the longitudinal emittance.

At injection. and during the cooling process, there is a strong coupling between the longitudinal and transverse motion in this channel. Particles at a large transverse amplitude tend to have large synchrotron motions because pathlengths depend on the betatron motion or the transverse amplitude. The synchrotron oscillation tune in such channel is ill-defined: as the stochastic processes (energy loss straggling while loosing energy in the absorber and, more importantly, scattering in the absorber or the foils) move the particles suddenly in a slightly different location in phase space. As a result, one can only quote a broad range for the synchrotron wave length, of about 5 to $15 \mathrm{~m}$ This is also the scale at which the correlation between the transverse amplitude and the momentum fades away, concurrent with the longitudinal emittance growth. The loss of this correlation itself implies a emittance growth, which has not been taken into account quoting the emittance at injection.

The result presented in figure 1 has been obtained for $50 \mu \mathrm{m}$ thick stainless steel $\mathrm{LH}_{2}$ windows. This is a rather conservative design. We have studied the cooling performance of the channel varying the thickness and material for these window, and observed a degradation of 5 to 10 $\%$ in the ratio the initial/final $6 \mathrm{D}$ emittance per additional $25 \mu \mathrm{m}$ of stainless steel. Titanium or Aluminium could also be used, but does not provide significantly better performance, because such window must be slightly thicker. Note that these windows can be made hemispherical, which gives enhanced mechanical strength without compromising the beam dynamics.

Another uncertainty in this calculation is due to the imperfect simulation of multiple scattering in Geant, (or similar packages), via the Moliere formalism [7]. The Moliere theory has been verified in numerous situation. However, for light materials, the uncertainty in the effective atomic screening angle could lead to uncertainties of a few \%. But, more relevant to our application, the Moliere formalism no longer holds for very thin absorbers where the "effective number of collisions" is small [8]. The $L H_{2}$ vessel must be divided into such thin layers while tracking, because of the betatron motion of the particles. The average scattering angle in small steps becomes comparable to the angle due to the Larmor precession in the high magnetic field. In $\mathrm{LH}_{2}$, such steps are of the order of a few microns, with angle of tens of $\mu \mathrm{rad}$. A detailed simulation, (few $\mu \mathrm{m}$ steps !) indicates that the Moliere approximation overestimates the radial displacement of the muon track in the absorber, leading to an over estimate of the beam reheating process. Based on a preliminary result, the ratio on the initial to final 2-D emittance could be $30 \%$ to $50 \%$ larger than estimated via the Moliere formalism.

Finally, if this channel remains a single-pass device (i.e., not embedded in a cooling ring), one could consider slightly increasing (few \% per section) the magnetic field in order to keep the cooling rate optimal.
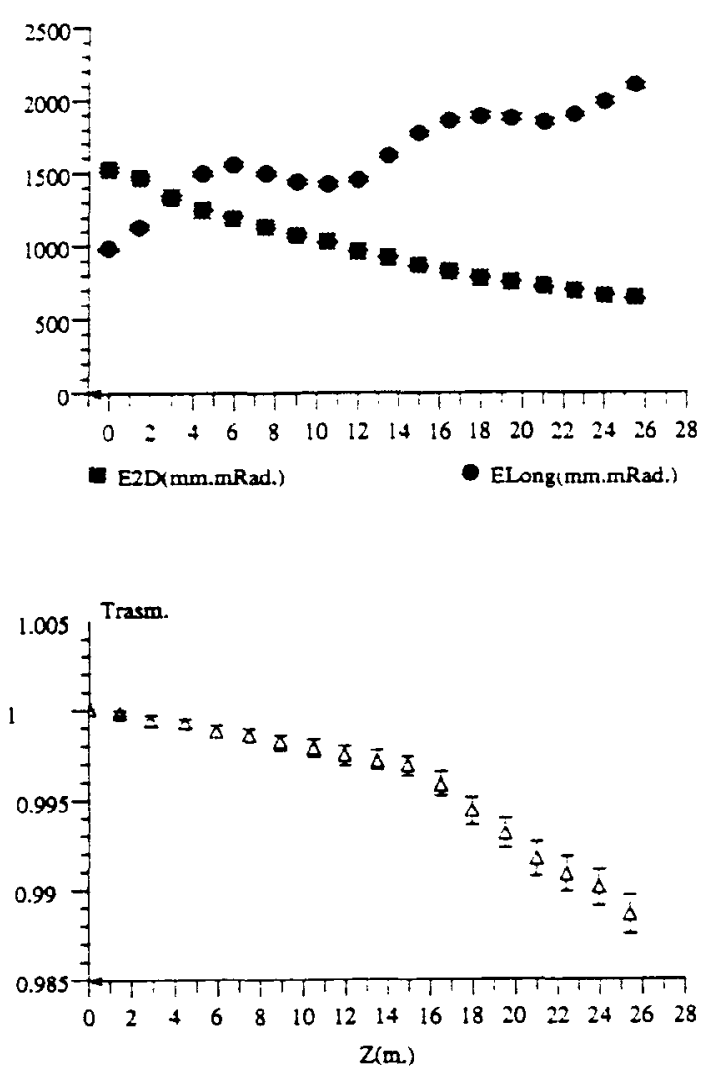

Figure 2: Top: the 2-dim. transverse emittance (squares) and the longindinal emittance (dots) versus the distance along the channel, showing a net 6-dim. cooling. Also shown (bottom) is the transmission, (The decay loss of about $2 . \%$ is not included).

\section{REFERENCES}

[1] Status of the Muon Collider Research and Development and Future Plans submitted to Phys. Rev., BNL-65-623, Fermilab -PUB-98/179. LBNL-41935.

[2] G. I. Budker and A. N. Skrinsky, Electron cooling and new possibilities in elementary particle physics, Sov. Phys. L'sp. 21277 (1978)

[3] H. Kirk, Parmela Modeling of Alternate Solenoids, presented at the mini-workshop on Ionization Cooling (BNL. 1998).

[4] R. C. Femow, ICOOL: A Simulation Code for Ionization Cooling of Muon Beams, these proceedings. THP31.

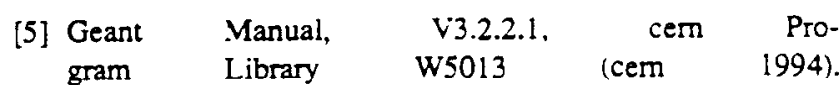
htp://wwwinfo.cem.ch/asdoc'geanthtmi3/geantall.html

[6] Convention taken from An introduction to the physics of high energy accelerators, John Wiley \& Sons, 1993, by D.A. Edwards and M.J. Syphers, p. 33.

[7] See for instance C. Caso et al Eur. Phys. J. C 3 1-794 (1998). page 146.

[8] H. A. Bethe Phys. Rev. 891256 (1953) 